\title{
Announcing IDA-99
}

\author{
Call for Papers: Third International Symposium on Intelligent Data Analysis Center for \\ Mathematics and Computer Science, Amsterdam, The Netherlands 9th-11th August 1999
}

IDA-99 will take place in Amsterdam from 9th to 11th August 1999, and is organized by Leiden University in cooperation with AAAI and NVKI. It will consist of a stimulating program of tutorials, invited talks by leading international experts in intelligent data analysis, contributed papers, poster sessions, and an exciting social program.

We plan to have a special issue of the Intelligent Data Analysis journal with extended versions of a number of papers presented during the symposium.

\section{Objective}

For many years the intersection of computing and data analysis contained menu-based statistics packages and not much else. Recently, statisticians have embraced computing, computer scientists are using statistical theories and methods, and researchers in all corners are inventing algorithms to find structure in vast online datasets. Data analysts now have access to tools for exploratory data analysis, decision tree induction, causal induction, function finding, constructing customized reference distributions, and visualization; there are intelligent assistants to advise on matters of design and analysis. There are tools for traditional, relatively small samples and also for enormous datasets. In all, the scope for probing data in new and penetrating ways has never been so exciting.

Our aim is for IDA-99 to bring together a wide variety of researchers concerned with extracting knowledge from data, including people from statistics, machine learning, neural networks, computer science, pattern recognition, database management, and other areas. The strategies adopted by people from these areas are often different, and a synergy results if this is recognized. IDA-99 is intended to stimulate interaction between these different areas, so that more powerful tools emerge for extracting knowledge from data and a better understanding is developed of the process of intelligent data analysis.

It is the third symposium on Intelligent Data Analysis after the successful symposia Intelligent Data Analysis 97 (http://www.dcs.bbk.ac.uk/ida97.html/) and Intelligent Data Analysis 95. 


\section{Topics}

Contributed papers are invited on any relevant topic, including, but not restricted to:

\section{APPLICATION AND TOOLS:}

- analysis of different kinds of data (e.g., censored, temporal etc)

- applications (e.g., commerce, engineering, finance, legal, manufacturing, medicine, public policy, science)

- assistants, intelligent agents for data analysis

- evaluation of IDA systems

- human-computer interaction in IDA

- IDA systems and tools

- information extraction, information retrieval

\section{THEORY AND GENERAL PRINCIPLES:}

- analysis of IDA algorithms

- classification, projection, regression, optimization, clustering

- data cleaning

- data pre-processing

- experiment design

- model specification, selection, estimation

- reasoning under uncertainty

- search

- statistical strategy

- uncertainty and noise in data

\section{ALGORITHMS AND TECHNIQUES:}

- Bayesian inference and influence diagrams

- bootstrap and randomization

- causal modeling

- data mining

- decision analysis

- exploratory data analysis

- fuzzy, neural and evolutionary appraoches

- knowledge-based analysis

- machine learning

- statistical pattern recognition

- visualization 


\section{Submissions}

Participants who wish to present a paper are requested to submit a manuscript, not exceeding 10 single-spaced pages. We strongly encourage authors to format their manuscript using Springer-Verlag's Advice to Authors (http://www.springer.de/comp/lncs/authors.html) for the Preparation of Contributions to LNCS Proceedings. This submission format is identical to the one for the final camera-ready copy of accepted papers. In addition, we request a separate page detailing the paper title, authors names, postal and email addresses, phone and fax numbers. Email submissions in Postscript form are encouraged. Otherwise, five hard copies of the manuscripts should be submitted. Submissions should be sent to the IDA-99 Program Chair either electronically to: ida99@wi.leidenuniv.nl or by hard copy to:

Prof. dr J.N. Kok, Department of Computer Science, Leiden University, P.O. Box 9512, 2300 RA Leiden, The Netherlands

The address for courier services is:

Prof. dr J.N. Kok, Department of Computer Science, Leiden University, Niels Bohrweg 1, 2333 CA Leiden, The Netherlands

\section{Important Dates}

- February 1st, 1999 Deadline for submitting papers

- April 15th, 1999 Notification of acceptance

- May 15th, 1999 Deadline for submission of final papers

\section{Review}

All submissions will be reviewed on the basis of relevance, originality, significance, soundness and clarity. At least two referees will review each submission independently and final decisions will be made by program chairs, in consultation with relevant reviewers.

\section{Publications}

The proceedings will be published in the Lecture Notes in Computer Science series of Springer (http://www.springer.de/comp/lncs/). The proceedings of Intelligent Data Analysis 97 appeared in this series as LNCS 1280 (http://www.springer.de/comp/lncs/volumes/1280.htm).

\section{Location}

The symposium will use the facilities of the Center for Mathematics and Computer Science in Amsterdam (CWI - http://www.cwi.nl/). There is an auditorium for more than 200 participants and several other rooms for parallel sessions. CWI is situated on the Wetenschappelijk Centrum 
Watergraafsmeer (WCW) campus in the eastern part of Amsterdam. Instructions about how to get to CWI can be found at: http://www.cwi.nl/cwi/about/directions.html On the campus there are several other research institutes and parts of the University of Amsterdam (http://www.uva.nl/english).

\title{
Social Event
}

For the social event we are thinking about a boat trip through the canals of Amsterdam, with a special dinner in the centre of the city. We will provide each participant with a "social package", including a list of restaurants, bars, maps of town (http://www.channels.nl/themap.html), public transport information, timetables of trains (http://www.ns.nl/), etc. There are special boats and trams that circle along the touristic attractions of Amsterdam and hence it will be easy for the participants to find their way. Further information can be found in the The Internet Guide to Amsterdam (http://www.cwi.nl/ steven/amsterdam.html), panoramic pictures are also available (http://www.cwi.nl/ behr/PanoramaUK/Panorama.html).

\section{Exhibitions}

IDA-99 welcomes demonstrations of software and publications related to intelligent data analysis.

\section{IDA-99 Organization}

\author{
General Chair: $\quad$ David Hand, Open University, UK \\ Program Chair: $\quad$ Joost Kok, Leiden University, The Netherlands \\ Program Co-Chairs: Michael Berthold, University of California, USA \\ Doug Fisher, Vanderbilt University
}

\section{Members:}

Niall Adams, Open University, UK

Pieter Adriaans, Syllogic, The Netherlands

Russell Almond, Research Statistics Group, USA

Thomas Baeck, Informatik Centrum Dortmund, Germany

Riccardo Bellazzi, University of Pavia, Italy

Paul Cohen, University of Massachusetts, USA

Paul Darius, Leuven University, Belgium

Tom Dietterich, Oregon State University, USA

Gerard van den Eijkel, Delft University of Technology, The Netherlands

Fazel Famili, National Research Council, Canada

Karl Froeschl, Univ of Vienna, Austria 
Linda van der Gaag, Utrecht University, The Netherlands

Alex Gammerman, Royal Holloway London, UK

Jaap van den Herik, University Maastricht, The Netherlands

Larry Hunter, National Library of Medicine, USA

David Jensen, University of Massachusetts, USA

Bert Kappen, Nijmegen University, The Netherlands

Hans Lenz, Free University of Berlin, Germany

Frank Klawonn, University of Applied Sciences Emden, Germany

Bing Liu, National University, Singapore

Xiaohui Liu, Birkbeck College, UK

David Madigan, University of Washington, USA

Heikki Mannila, Helsinki University, Finland

Wayne Oldford, Waterloo, Canada

Erkki Oja, Helsinki University of Technology, Finland

Albert Prat, Technical University of Catalunya, Spain

Luc de Raedt, KU Leuven, Belgium

Rosanna Schiavo, University of Venice, Italy

Jude Shavlik, University of Wisconsin, USA

Roberta Siciliano, University of Naples, Italy

Arno Siebes, Center for Mathematics and Computer Science, The Netherlands

Rosaria Silipo, International Computer Science Institute, USA

Floor Verdenius, ATO-DLO, The Netherlands

Stefan Wrobel, GMD, Germany

Jan Zytkow, Wichita State University, USA

(To be extended)

\section{Inquiries}

Latest information regarding IDA-99 will be available on the World Wide Web Server of the Leiden Institute for Advanced Computer Science: http://www.wi.leidenuniv.nl/rida99/ 